\title{
Can Vitamin D Supplementation Reduce Risk of Recurrence of Pneumonia in Under-Five Children?
}

\author{
B. Kiran Kumar ${ }^{1} \cdot$ Rakesh Lodha $^{1}$
}

Received: 16 October 2019 / Accepted: 16 October 2019 / Published online: 8 November 2019

(C) Dr. K C Chaudhuri Foundation 2019

Pneumonia continues to be an important cause of mortality in under-five children [1]. There has been considerable interest in use of Vitamin D in preventing childhood pneumonia and in improving the outcomes of children with pneumonia. Vitamin $\mathrm{D}$, a fat-soluble vitamin, apart from calcium and phosphate homeostasis, has multiple effects. It modulates innate and acquired immunity and regulates inflammatory cascade. Many of the immune cells express Vitamin D receptors. Vitamin D regulates macrophage and dendritic cell activities and toll like receptor mediated response in neutrophils. Vitamin D induces expression of cathelicidin and $\beta$-defensin which have chemotactic and toxin neutralization properties [2]. Association between Vitamin D deficiency and increased risk of pneumonia is well studied [3, 4]. Also, association between Vitamin D deficiency and severity of lower respiratory tract infection has been recognized [5]. However, these associations and possible biologic basis for role of preventing and improving treatment outcomes of childhood pneumonia have not been supported by clinical trials.

Yakoob et al., evaluating the role of Vitamin D in preventing infections in their systematic review, included two trials on childhood pneumonia and found that there was no difference in the incidence of first episode of pneumonia in children supplemented with Vitamin D [6]. For use of Vitamin $\mathrm{D}$ as an adjunct to antibiotic treatment of childhood pneumonia, Das et al. in their systematic review reported that the effects of Vitamin D supplementation in childhood pneumonia on outcomes, including time to resolution of acute illness,

Rakesh Lodha

rlodha1661@gmail.com

1 Division of Pediatric Pulmonology and Intensive Care, Department of Pediatrics, All India Institute of Medical Sciences, New Delhi, India mortality rate and resolution of fever, were inconclusive and the quality of evidence was low to very low [7].

Some children with pneumonia may be at risk of recurrence of pneumonia. Researchers have also evaluated the role of Vitamin D in reducing this risk of recurrence.

In the current issue, Singh et al. report the results of randomized controlled trial evaluating the role of Vitamin D supplementation of 3,00,000 IU quarterly doses for 4 doses in the prevention of recurrence of pneumonia in under-five children and reported no statistically significant beneficial effect [8]. The mean lower respiratory tract infection (LRTI) episodes were slightly less in the intervention group, although the mean duration of LRTI episodes was longer in the intervention group. Also, they observed no benefit in the mean upper respiratory tract infection (URTI) episodes, complication rate and severity of pneumonia. The follow up period was sufficiently long. But the study has few limitations. It was from a small cohort of children (the sample size calculation was not provided). Also, as mentioned by the authors, follow up Vitamin D levels were not available [8].

In a similar community-based study done in Kabul enrolling 3046 children, Vitamin D was administered to young infants quarterly at a dose of 1,00,000 IU; there was no difference in incidence of first or only episode of pneumonia although incidence of repeated episodes of pneumonia was higher in the Vitamin D group [9]. Gupta et al., in a doubleblind randomized trial found that Vitamin D supplementation did not reduce the risk of recurrence of pneumonia in 6-mo follow up period [10].

While there have been differences in the dosing schedules of different studies, current evidence does not support the use of Vitamin D in prevention or treatment of childhood pneumonia; neither the immediate outcomes nor the risk of recurrence of pneumonia seem to benefit from Vitamin D supplementation. 


\section{Compliance with Ethical Standards}

Conflict of Interest All authors declare no conflicts of interest.

\section{References}

1. Liu L, Oza S, Hogan D, et al. Global, regional, and national causes of under-5 mortality in 2000-15: an updated systematic analysis with implications for the sustainable development goals. Lancet. 2016;388:3027-35.

2. Esposito $\mathrm{S}$, Lelii M. Vitamin $\mathrm{D}$ and respiratory tract infections in childhood. BMC Infect Dis. 2015;15:487.

3. Karatekin G, Kaya A, Salihoglu O, et al. Association of subclinical vitamin D deficiency in newborns with acute lower respiratory infection and their mothers. Eur J Clin Nutr. 2009;63:473-7.

4. Roth DE, Shah R, Black RE, Baqui AH. Vitamin D status and acute lower respiratory infection in early childhood in Sylhet, Bangladesh. Acta Paediatr. 2010;99:389-93.

5. Jat KR. Vitamin D deficiency and lower respiratory tract infections in children: a systematic review and meta-analysis of observational studies. Trop Doct. 2017;47:77-84.
6. Yakoob MY, Salam RA, Khan FR, Bhutta ZA. Vitamin D supplementation for preventing infections in children under five years of age. Cochrane Database Syst Rev. 2016;11:CD008824.

7. Das RR, Singh M, Naik SS. Vitamin D as an adjunct to antibiotics for the treatment of acute childhood pneumonia. Cochrane Database Syst Rev [Internet]. 2018;7:CD011597.

8. Singh N, Kamble D, Mahantshetti NS. Effect of vitamin D supplementation in the prevention of recurrent pneumonia in under-five children. Indian J Pediatr. 2019. https://doi.org/10.1007/s12098019-03025-z.

9. Manaseki-Holland S, Maroof Z, Bruce J, et al. Effect on the incidence of pneumonia of vitamin D supplementation by quarterly bolus dose to infants in Kabul: a randomized controlled superiority trial. Lancet. 2012;379:1419-27.

10. Gupta P, Dewan P, Shah D, et al. Vitamin D supplementation for treatment and prevention of pneumonia in under-five children: a randomized double-blind placebo controlled trial. Indian Pediatr. 2016;53:967-76.

Publisher's Note Springer Nature remains neutral with regard to jurisdictional claims in published maps and institutional affiliations. 